\title{
Human stem cell models of disease and the prognosis of academic medicine
}

\author{
Rickie Patani is Professor of Human Stem Cells and Regenerative Neurology at University College London and The \\ Francis Crick Institute, and is a consultant neurologist at The National Hospital for Neurology, Queen Square, London. \\ He has over a decade of experience using human stem cell models of neurodegeneration, and his research contributions \\ have been recognized by the International Paulo Gontijo Award in Medicine and the International 3Rs prize.
}

\section{Rickie Patani}

$\mathrm{T}$ he first time I looked down a microscope and saw human brain tissue, I was mesmerized by a galaxy of complexity_ _ it was just miraculous and captivating. I trained in clinical medicine at Imperial College London, completing an intercalated degree in molecular neuroscience. The first neurology patient I reviewed soon after qualifying from medical school presented with progressive weakness, swallowing difficulties and shortness of breath over 18 months. I presented his case to the senior general physician on call, who said "I agree this is ALS. I would give him a cigar, close the curtains and move on to the next patient...there is little more you can do here." Those words were burned into my memory and really confirmed my becoming a neurologist_- it seemed entirely unacceptable that we could not treat amyotrophic lateral sclerosis (ALS) effectively. I soon realized what was so desperately needed for ALS (and indeed most other neurodegenerative disorders): the discovery of molecular mechanisms of disease in order to guide therapeutic strategy. I then spent much of my personal time researching what was being done in laboratories and clinics to understand this disease. I came to appreciate that, despite being undeniably valuable across both basic neuroscience and applied neuroscience, model organisms had not delivered an impactful therapy for ALS. Additionally, post-mortem tissue captured mainly an advanced state of disease and therefore did not allow elucidation of the primacy of molecular pathogenic events. It occurred to me that a human experimental model was needed to complement, but not replace, existing approaches in order to capture the clinical pathophysiological state with more fidelity and precision — this is when my passion for academic medicine was ignited.

I started on this journey by securing a higher training post in clinical neurology and undertaking a $\mathrm{PhD}$ in human stem cell

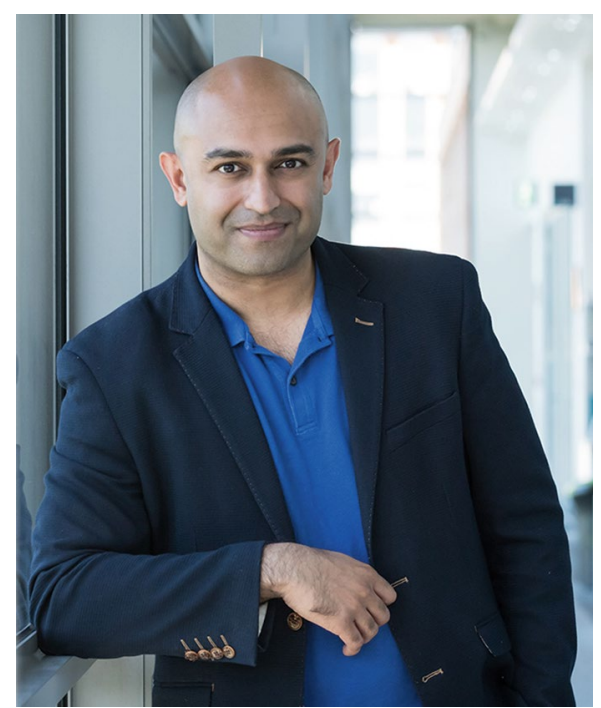

Credit: The Francis Crick institute | Dave Guttridge

neurobiology (funded by the Wellcome Trust) at Cambridge University, where I also stayed on for postdoctoral work. I became fascinated by the ability to direct the differentiation of human pluripotent stem cells into regionally specified neurons and glia by harnessing developmental insights. I was excited at the prospect of modeling a patient's disease using stem cells, which bypassed the need for artificial overexpression and/or knockdown, permitted time-resolved molecular interrogation of cell type-specific pathogenic events and advanced us towards personalized medicine in one fell swoop. I then secured a Wellcome Trust Intermediate Clinical Fellowship and chose to move to University College London Queen Square Institute of Neurology to start my laboratory during the completion of my higher clinical training. Over this time, we made insights into which molecular pathogenic events occurred in ALS, and in which cell types and when, by developing highly enriched populations of functional human motor neurons and astrocytes. We uncovered an interesting posttranscriptional phenomenon called 'aberrant intron retention' in ALS, which led to the discovery of two new molecular hallmarks in this disease. I then secured an MRC Senior Clinical Fellowship to examine these potential post-transcriptional pathomechanisms in human stem cell models of ALS. My group is now fully based at the Francis Crick Institute.

I am a physician-scientist, dually trained in clinical medicine (neurology) and basic science (stem cell biology). This has been a long and challenging journey but also an immensely rewarding one. I find it highly motivating to see patients in my clinic; it inspires our research in the lab and keeps our core goal_-i.e., patient benefitfirmly in mind. Indeed, this crucial integrative role between clinical medicine and basic science is a fundamental driver of translational science. This is exemplified by the fact that over a third of the recipients of the Nobel Prize in Physiology or Medicine hold medical degrees. However, the number of clinician scientists working in the UK is declining. This career path seems to be both increasingly competitive and disincentivized. I strongly believe that we (society, governments, research institutions, hospital trusts and individual clinician scientists) can and must do more to incentivize and inspire talented clinicians to follow this unique pathway, helping to improve translational science and finding answers to pertinent research questions for ultimate patient benefit. By doing this, we can move closer to finding cures and defeating devastating diseases such as ALS.

\section{Rickie Patani}

Human Stem Cells and Neurodegeneration Laboratory, The Francis Crick Institute, University College London, London, UK.

e-mail: rickie.patani@crick.ac.uk

Published online: 9 April 2020 https://oi.org/10.1038/s41591-020-0814-7 